\title{
Hybridization-Promoted and Cytidine-Selective Activation for Cross-Linking with the Use of 2-Amino-6-Vinylpurine Derivatives
}

\section{Takeshi Kawasaki, Fumi Nagatsugi, Md. Monsur Ali, Minoru Maeda, Kumiko Sugiyama, Kenji Hori and Shigeki Sasaki*}

\section{Experimental}

General. All melting points are uncorrected. The $1 \mathrm{H}$ NMR and DQF-COSY spectra were recorded at $400 \mathrm{MHz}$ and $600 \mathrm{MHz}$, respectively, using tetramethylsilane (TMS) as an internal standard. The ${ }^{31} \mathrm{P}$ and ${ }^{13} \mathrm{C}$ NMR spectra were recorded at $162 \mathrm{MHz}$ using phosphoric acid as an internal standard and at $125 \mathrm{MHz}$ using $\mathrm{CDCl} 3$ as an internal standard, respectively. IR spectra were recorded in a film. FABMS and High-resolution FABMS spectra were measured by positive mode using 4-nitrobenzylalchol as the matrix. MALDI-TOF mass spectra were measured by negative mode using the laser at $337 \mathrm{~nm}$ and 3-hydroxypicolinic acid as the matrix. All solvents were distilled prior to use from an appropriate drying agent. Column chromatography was performed with silica gel (70-230 mesh). Thin-layer chromatography (TLC) was carried out with silica gel plates $(0.25$ $\mathrm{mm}$ ). HPLC was performed on ODS columns monitoring at $254 \mathrm{~nm}$. Densitometric analysis of the gel was carried out on $20 \%$ denaturing polyacrylamide gel plates. All enzymes and [g-32P]ATP $(4500 \mathrm{Ci} / \mathrm{mmol})$ were obtained from commercial sources. 
$y=t=43,61$

Pulue sesuence: Ispol

soivery cicia

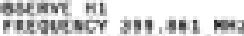

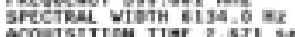

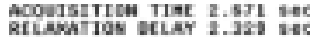

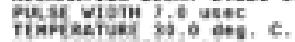

tonteririos

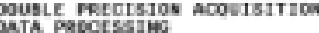

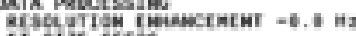

retai Acoifsifren Tros 1 aireute
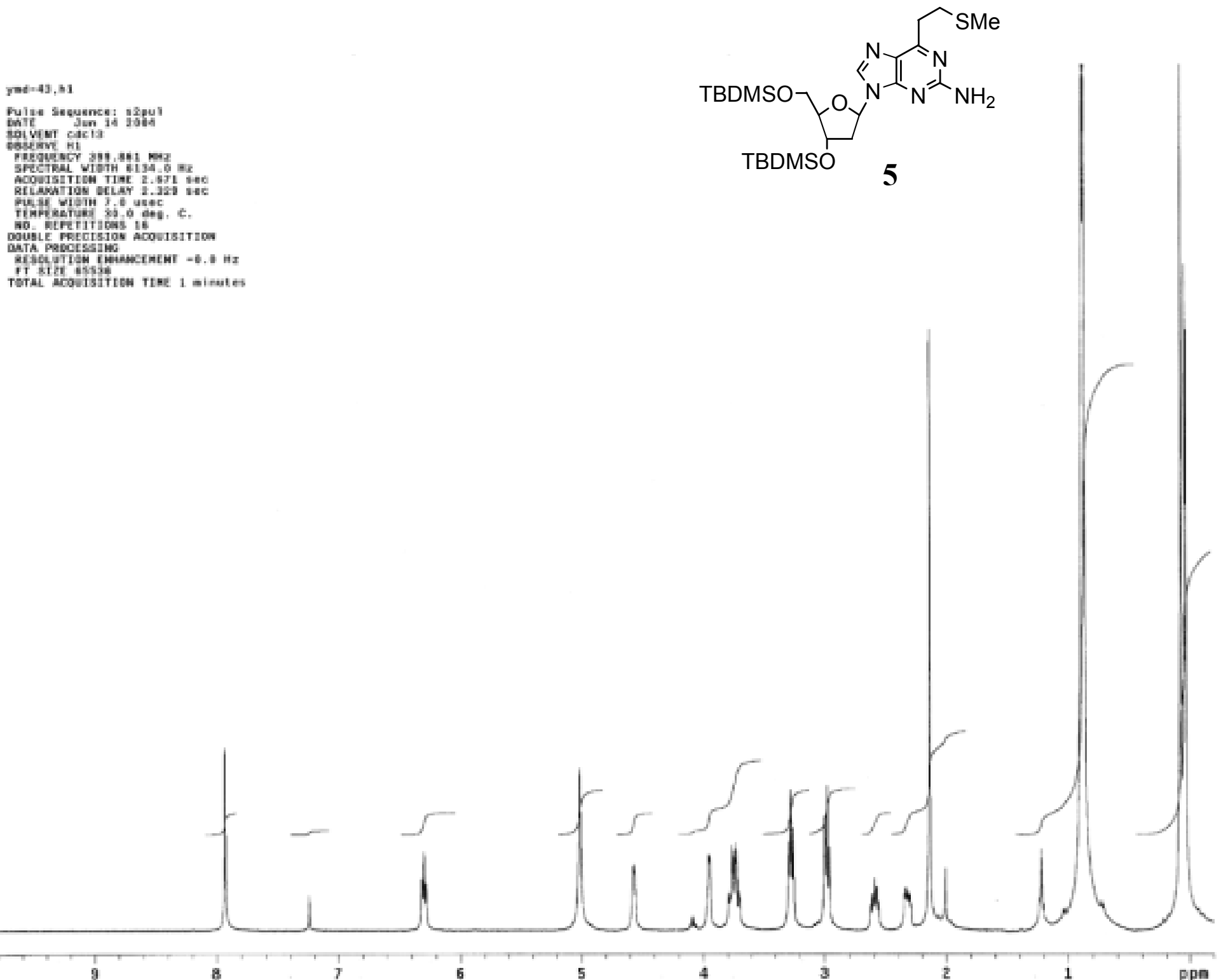
roo-es.6i160et

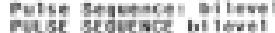

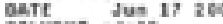

ótivent cecis

reroutucr 100 .55 me

ecqutsitaos

nciecartem ofiar 2.143 ite

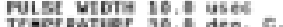

tcisuric ni

Hich povk sin

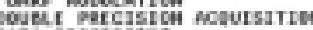

itut eataotution $5.6 \mathrm{~Hz}$

Toth acturstios rime s mimates

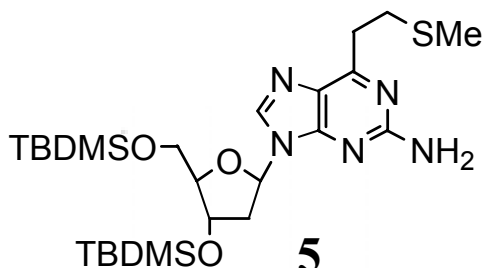

5

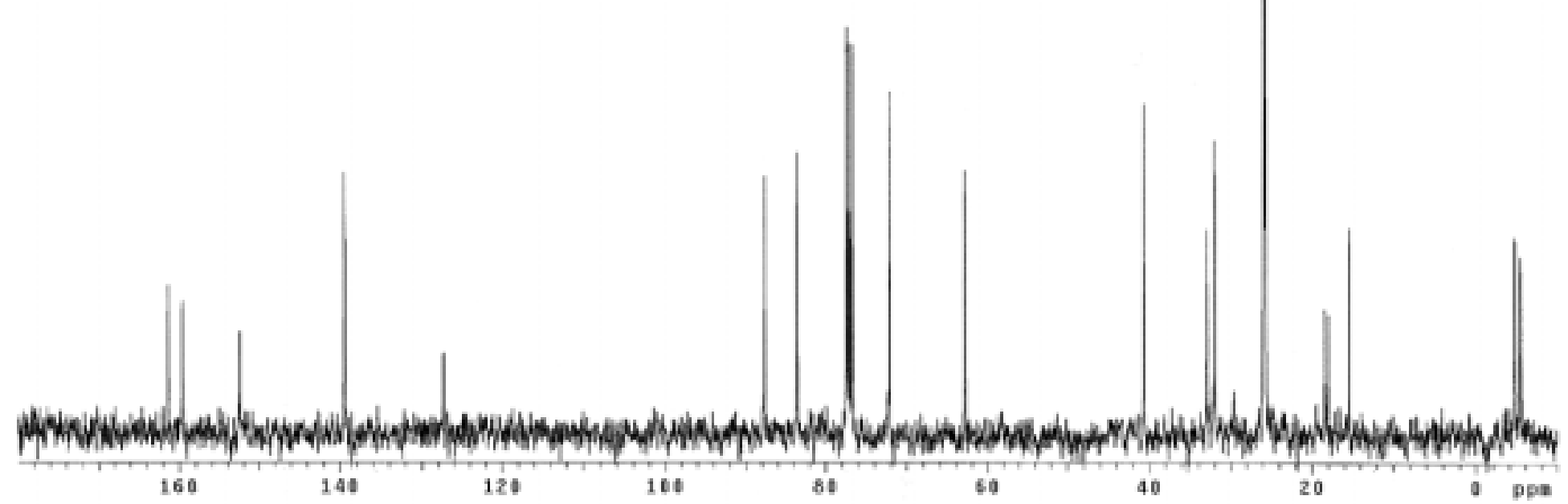


yed-47, hi

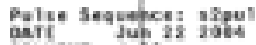

salvent cecis

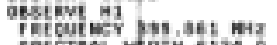

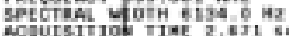

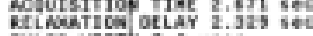

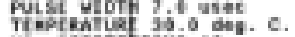

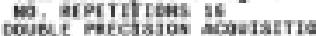

(strias

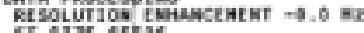

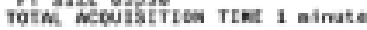

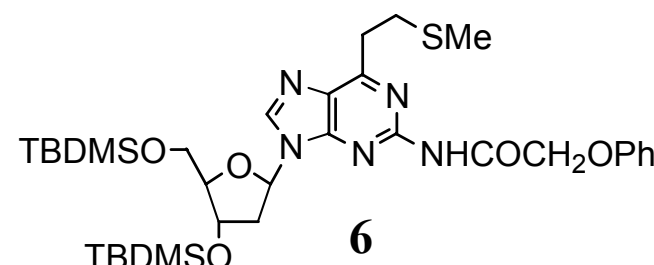

TBDMSO

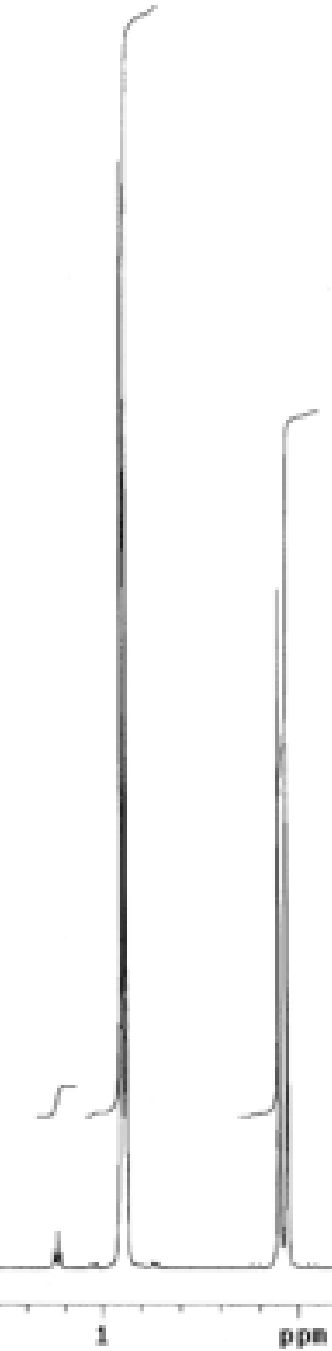




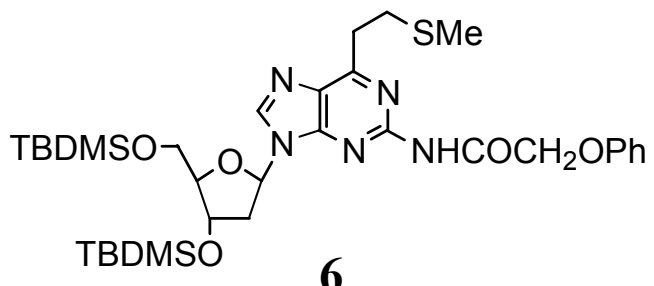

no_4s, bsleve:

Tules Sequebeer bilerel pular sajubica bijavel

DNTS Oet 42034

GoLvers cdei.

rovguner 125 - $694 \mathrm{keq}$

agectal viots 28543 .

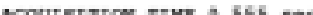

Megur

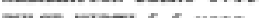

Ther wism 6.6 usec

wo. aseseresegs ajob

Wo. Mkntritsoss gab

Deoourhe ni

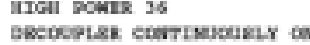

EWCOEPLAk Cantian

Gasp MoOteartos

pedike paberata

DATA Fockserise

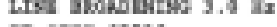

it aI8E 65536

TOTaL Aogurersion TrMe 53 minute.

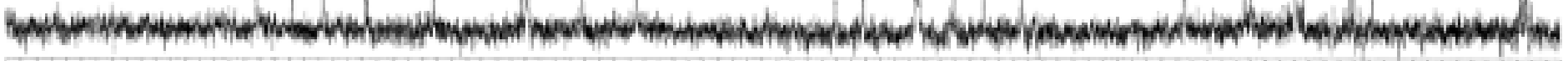


soiver ows

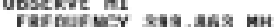

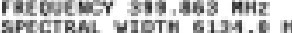
acoursitios rine $2.671 \mathrm{sec}$

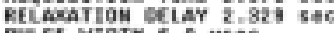

Tente

40.

coubl parcision hoquisitron

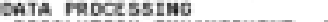

Resoturion enhencenesr $-4.0 \mathrm{~Hz}$

ToTaL Acouisition trat 1 minutes

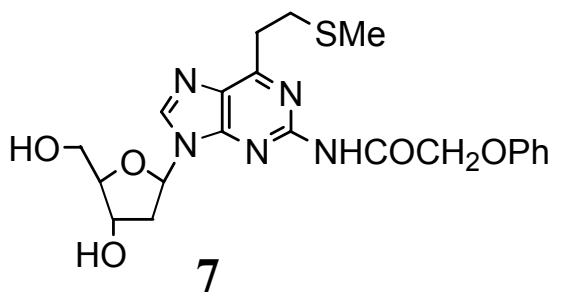




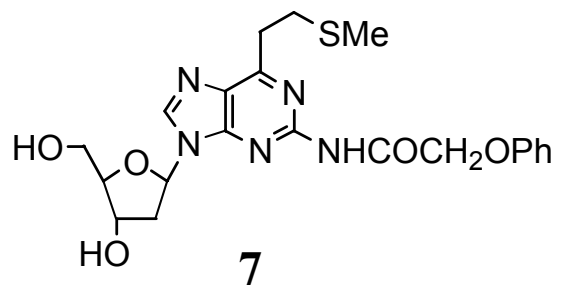

hulas tequence: dept

DULSE sagunNer dept.

Buse aep 20E4

cotrant tose

Fospaner $125.685 \mathrm{kE}$

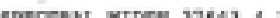

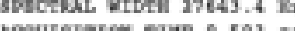

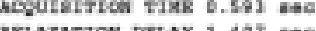

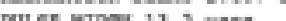

hular wipha 13.2 unes

Teaskerike 30.0 ded.

so. Mrvents

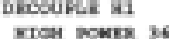

rTen NowE 36

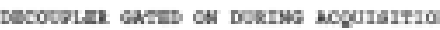

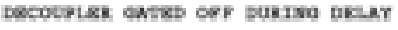

ank moochertos

DoukL pReriatos neguiartios

DaTh Fockstrag

\section{pe 313565536}

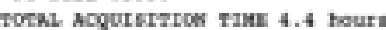

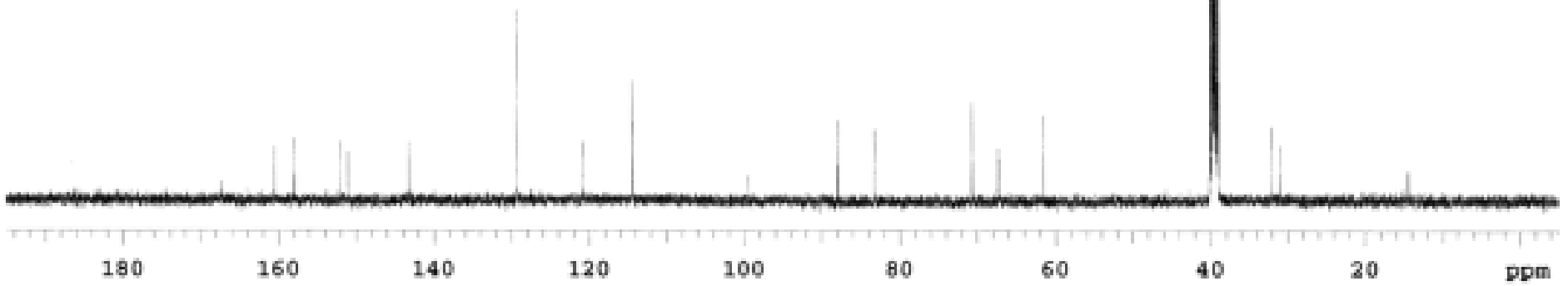




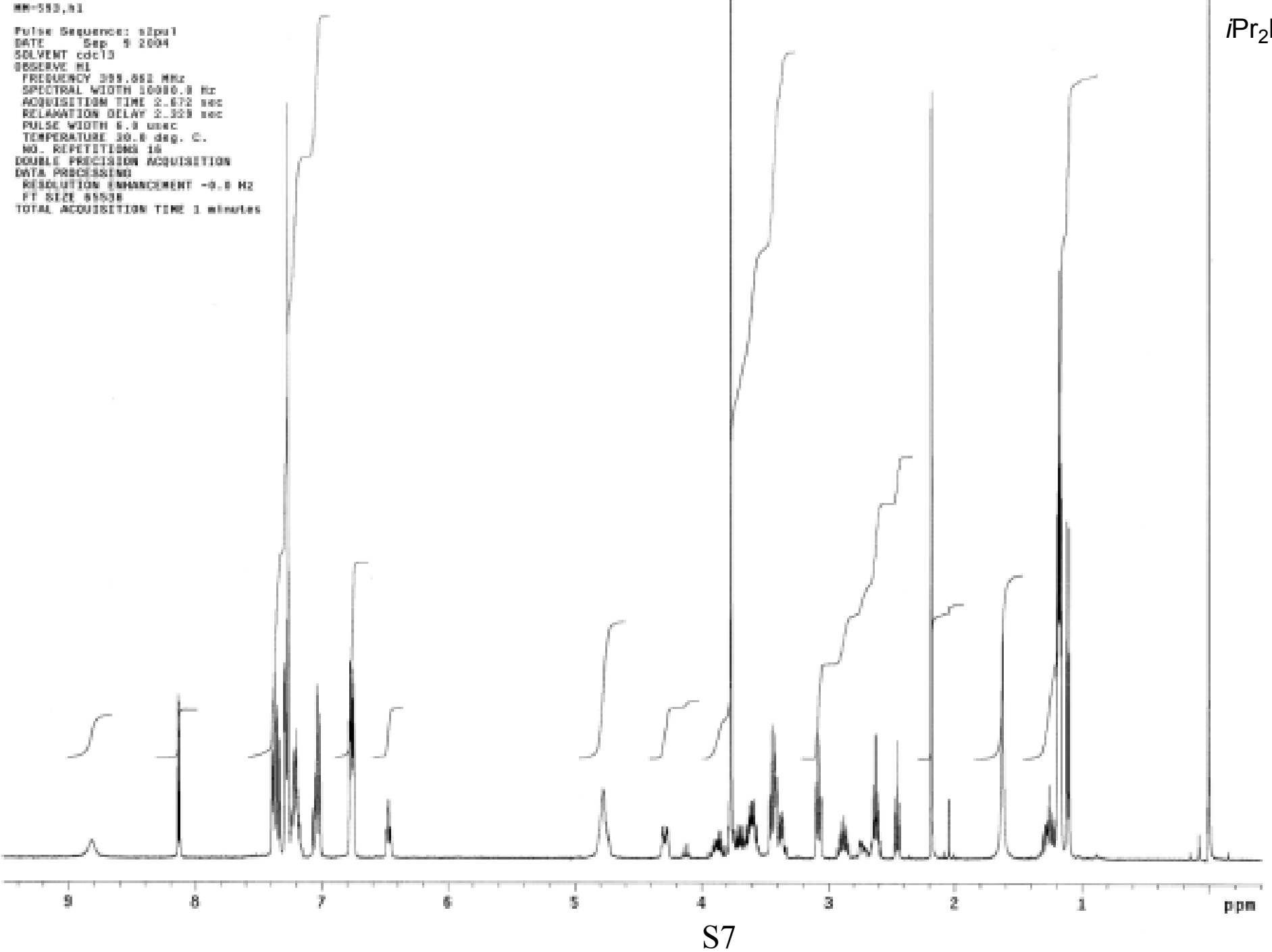


$\rightarrow \mathrm{si}$

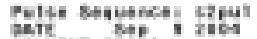

setvest ciets

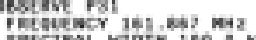

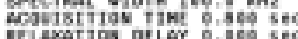

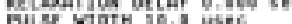

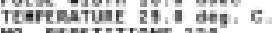

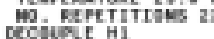

Hich fouta 3

otcoupies costrsyousty a

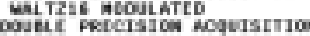

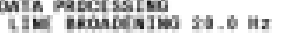

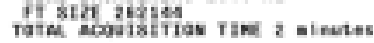

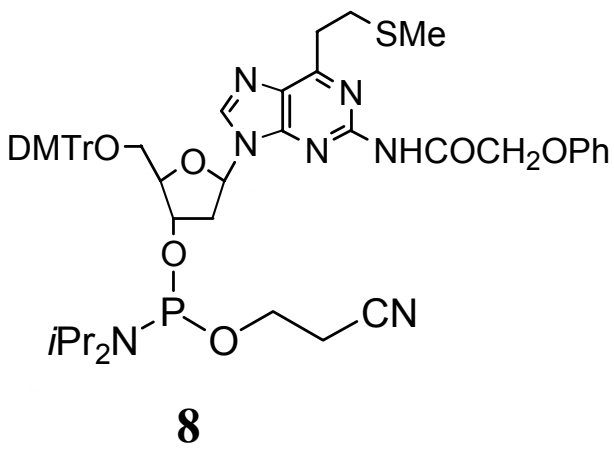

8

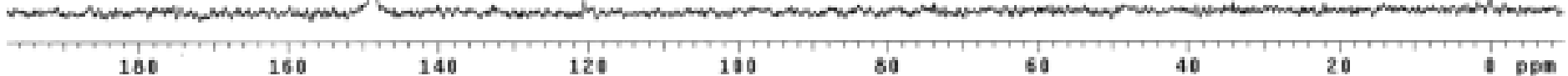

